This document is the Accepted Manuscript version of a Published Work that appeared in final form in Analytical Chemistry, copyright (C) American Chemical Society after peer review and technical editing by the publisher. To access the final edited and published work see http://dx.doi.org/10.1021/ac034473a

\title{
Calibration of ion effective temperatures achieved by resonant activation in a quadrupole ion trap
}

\author{
Valérie Gabelica ${ }^{*}+$, Michael Karas ${ }^{\dagger}$, Edwin De Pauw ${ }^{\ddagger}$
}

Institüt für Pharmazeutische Chemie, Johann-Wolfgang Goethe Universität Frankfurt, Marie-Curie Str. 9-11, D-60439 Frankfurt am Main, Germany and Département de Chimie, Université de Liège, Bat. B6c, B-4000 Liège, Belgium

* corresponding author: v.gabelica@ulg.ac.be. Present address: Département de Chimie, Université de Liège, Bat. B6c, B-4000 Liège, Belgium.

RECEIVED DATE (to be automatically inserted after your manuscript is accepted if required according to the journal that you are submitting your paper to).

$\dagger \mathrm{J}-\mathrm{W}$ Goethe Universität Frankfurt

† Université de Liège 


\section{ABSTRACT.}

The present paper describes a calibration of the ion effective temperatures as a function of the resonant activation amplitude in a quadrupole ion trap mass spectrometer. MS/MS experiments are performed on leucine enkephalin $(\mathrm{M}+\mathrm{H})^{+}$, bradykinin $(\mathrm{M}+\mathrm{H})^{+},(\mathrm{M}+2 \mathrm{H})^{2+}$ and $(\mathrm{M}+3 \mathrm{H})^{3+}$, and ubiquitin $(\mathrm{M}+11 \mathrm{H})^{11+}$. For each amplitude, the effective temperature is calculated as the temperature that would give the same dissociation rate constant as the one observed, and is calculated using published Arrhenius parameters. The effective temperature is found to be linearly dependent on the activation amplitude on the range investigated. The dependence of the slope and of the intercept of the $T_{\text {eff }}=\mathrm{f}$ (amplitude) functions on the parent ion $\mathrm{m} / \mathrm{z}$ is examined and an equation is derived to calibrate the ion effective temperature between 365 and $600 \mathrm{~K}$. Below $365 \mathrm{~K}$, a deviation from linearity is expected. Above $600 \mathrm{~K}$, the validity of the equation will depend on whether the rapid energy exchange limit is still reached. Calculating backwards the Arrhenius parameters from the measured dissociation rates using this calibration shows an excellent agreement with the published values. The calibration can therefore be used to determine Arrhenius activation parameters from dissociation kinetics under resonant activation in quadrupole ion trap mass spectrometers. 


\section{INTRODUCTION}

Electrospray ionization (ESI) has become a powerful technique for the transfer a wide variety of biomolecule ions (peptides, proteins, DNA,...) into mass spectrometers ${ }^{1-3}$. Of particular interest is the ability of ESI to transfer intact non-covalent complexes from native-like solutions to the gas phase ${ }^{4-10}$. Mass spectrometry offers a unique opportunity to study biologically-relevant molecules and complexes in the absence of solvent by a large panel of techniques, e.g. MS/MS by collisional ${ }^{6 ; 11-13}$ or thermal ${ }^{14 ; 15}$ activation, ion mobility techniques ${ }^{16 ; 17}$, hydrogen/deuterium exchange ${ }^{18 ; 19}$, or ion-ion reactions ${ }^{20}$.

By far, the most widely used technique is tandem mass spectrometry (MS/MS). The nature of the dissociation products and their relative intensities compared to the parent ion depend on the internal energy imparted to the parent ion, and on the activation enthalpy and the activation entropy ${ }^{21}$. Extracting energetic and dynamic information from MS/MS experiments therefore requires the characterization of the internal energy distribution of the parent ion, and of the time allowed for the dissociation $^{22 ; 23}$.

One such quantitative approach is the threshold-CID method, which has been applied to metal-ligand complexes ${ }^{23-27}$. As the dissociation is carried out ideally in single-collision conditions, both the internal energy distribution prior to collision and the energy imparted in the collision are well-defined. The determination of the energy thresholds for dissociation $\left(E_{0}\right)$ requires an RRKM modeling of the experimental data, and the kinetic shift must be taken into account. The method is therefore more difficult to apply as the size of the parent ion increases, due to the computation time required for the RRKM modeling.

A convenient way to achieve efficient collisional activation of larger molecules is to use multiple collisions $^{28}$, for example in quadrupole ion $\operatorname{traps}^{28}$ (resonant excitation), in FTICR mass spectrometers ${ }^{29}$ (e.g. by SORI-CAD), or in quadrupoles, as has been applied successfully to very large protein complexes $^{30 ; 31}$. It has been demonstrated that only a few collisions are required to achieve a Boltzmannlike internal energy distribution for the parent ion ${ }^{28 ; 32 ; 33}$. The internal energy distribution can therefore be characterized by a single parameter, called the effective temperature, which is the temperature of the 
Boltzmann internal energy distribution that would give the same spectrum as the actual distribution ${ }^{21}$. However the total amount of internal energy imparted by collisional activation (or the effective temperature) is still poorly defined and hard to predict in most cases, due to its dependence on many experimental parameters ${ }^{28 ; 34-37}$.

A special case where the internal energy distribution can be fully characterized is the case of thermal activation in the rapid energy exchange (REX) limit. The REX limit is attained when the activation and deactivation rates are much higher than the dissociation rate. If not perturbed by the dissociation and depleted at high energies, the internal energy distribution is therefore equal to the Boltzmann distribution at the temperature of the ion trapping cell walls. As a consequence, Arrhenius plots of the logarithm of the rate constant as a function of $1 / T$ gives access to energetic (through the activation energy $E_{a}$, which is equal to the dissociation threshold $E_{0}$ in the REX limit) and mechanistic information (through the pre-exponential factor $A$ ) for each dissociation channel.

Thermal dissociation in the REX limit can be realized be heating an FTICR trapping cell. Collisional energy transfer is not possible due to the low operating pressures, but temperature equilibration between the ion and the chamber walls is ensured by blackbody radiative activation and deactivation events. Blackbody radiation activation in FTICR-MS has been first demonstrated by McMahon and Dunbar on small molecules ${ }^{38-40}$. In those cases the REX limit is not attained and molecular modeling was necessary to relate the measured $E_{a}$ and the true threshold $E_{0}$. Later, Williams and co-workers have applied this method, called BIRD (blackbody infrared radiative dissociation) to larger biomolecules ${ }^{41-45}$, for which the REX limit is more easily reached, due to larger activation and deactivation rates ${ }^{46}$.

More recently, McLuckey and co-workers have realized thermal activation in a quadrupole ion trap instrument. In this case, energy transfer is ensured by multiple collisions with the heated bath gas (usually helium at a pressure of about $1 \mathrm{mTorr})^{36 ; 4748}$. It has been demonstrated that, in the absence of resonant activation, the ion dissociation rate was insensitive to the $\mathrm{rf}$ trapping potential, and therefore that the ion internal temperature was equal to the temperature of the quadrupole ion trapping cell. Moreover, detailed master equation modeling suggests that the REX conditions are satisfied for leucine 
enkephalin $(\mathrm{M}=556 \mathrm{Da})$ for dissociation rate constants $<1 \mathrm{~s}^{-1}$, and for bradykinin $(\mathrm{M}=1060 \mathrm{Da})$ for dissociation rate constants $<10 \mathrm{~s}^{-1} \cdot{ }^{36}$ Compared to the FTICR mass spectrometer, the larger dissociation rates that can be reached by heating the quadrupole ion trap while still being in the REX limit is due to the faster energy exchange between the ion and the environment by collisions with the relatively highpressure bath gas.

However, a common limitation of both (BIRD and QIT) thermal methods is the maximal experimentally achievable heating temperature, which is around $500 \mathrm{~K}$. As a consequence, only the lowest energy reaction pathways can be investigated by these methods. The second disadvantage of heating QIT or FTICR cells is that the achievement of a homogeneous, reproducible, and stable heating is experimentally demanding. These two reasons led us to investigate in more detail the possibilities for achieving thermal-like conditions by resonant activation in a quadrupole ion trap. The two requirements are (1) a calibration of the ion effective temperatures as a function of the activation conditions and (2) that the activation and deactivation rates are quick enough compared to the dissociation rate so as to be in the REX limit. If both requirements are met, Arrhenius activation parameters can be determined using resonant activation instead of thermal activation. The present paper focuses on the calibration of the effective temperatures.

The relationship between the ion internal temperature and the resonant activation parameters can be either predicted, or determined experimentally. Goeringer and McLuckey have used the kinetic theory of ion transport in gases and random walk simulations, using a simple forced damped harmonic oscillator approximation for the ion acceleration process, to model the evolution of the ion internal temperature with the resonant activation amplitude ${ }^{49 ; 50}$. The internal temperature is given by:

$$
T_{\text {eff }}=T_{\text {bath }}+\frac{m_{\text {gas }} C V^{2}}{3(m / z)^{2} \xi(T e f f)^{2}}
$$

where $T_{\text {bath }}$ is the temperature of the bath gas, $m_{\text {gas }}$ is the mass of the bath gas, $C$ is a collection of constants, $V$ is the activation amplitude, $m / z$ is the mass-to-charge ratio of the parent ion, and $\xi\left(T_{e f f}\right)$ is the reduced collision frequency, which depends on the ion-neutral interaction potential and on the 
internal energy. However the temperature scale is not absolute, due to approximations used in calculating $\xi\left(T_{e f f}\right)$. An experimental calibration is therefore required.

Such an experimental calibration has been reported for the small peptide leucine enkephalin $(\mathrm{M}=556$ $\mathrm{Da})^{51}$. The dissociation rate constants (from 1.6 to $66 \mathrm{~s}^{-1}$ ) were measured as a function of the resonant activation amplitude. As master equation modeling showed that the REX limit was not attained for dissociation rates larger than $1 \mathrm{~s}^{-1}$ (corresponding to effective temperatures $>525 \mathrm{~K}$ ), the calibration of the effective temperatures has been made by comparison between resonant activation and purely thermal activation (heating of the ion trap). The authors found a linear relationship between the ion effective temperature and the resonant excitation voltage $V$ over the range investigated ${ }^{51}$. Further studies of this nature are however needed with other thermometer ions, in order to address the ion $\mathrm{m} / \mathrm{z}$ dependency of the relationship between $T_{\text {eff }}$ and $V$. In the present article, we report the calibration of the effective temperature with leucine enkephalin and larger thermometer molecules (bradykinin and ubiquitin), for which Arrhenius activation parameters are available from the literature. 


\section{EXPERIMENTAL SECTION}

Leucine enkephalin $($ average mass $=556 \mathrm{Da})$, bradykinin $(1060 \mathrm{Da})$ and bovine ubiquitin $(8565 \mathrm{Da})$ have been purchased from Sigma-Aldrich (Taufkirchen, Germany). All samples were diluted to a concentration of $10^{-5} \mathrm{~mol} / \mathrm{L}$ in $0.1 \%$ aqueous acetic acid/acetonitrile $50 / 50(\mathrm{v} / \mathrm{v})$, and were infused in the electrospray source at the rate of $1.5 \mu \mathrm{L} / \mathrm{min}$.

Mass spectrometric experiments were performed on an LCQ electrospray quadrupole-ion trap instrument (ThermoFinnigan, San Jose, CA). The trap is filled with helium, and a constant pressure of $10^{-3}$ Torr is automatically maintained by the instrument. The room temperature was $22 \pm 1{ }^{\circ} \mathrm{C}$, and the ambient temperature inside the instrument (measured at the power supplies) was $25.6 \pm 0.4{ }^{\circ} \mathrm{C}$. Automatic data acquisition was processed by the software Xcalibur 1.1 (ThermoFinnigan). In our instrument, the actual amplitude in volts is related to the activation amplitude setting (in percent) by the equation:

$$
\operatorname{Ampl}(/ \mathrm{V})=\operatorname{Ampl}(/ \%) / 30 *[0.4+0.002(\mathrm{~m} / \mathrm{z})]
$$

This normalization is made to compensate the $\mathrm{m} / \mathrm{z}$ dependency of the fragmentation efficiency ${ }^{52}$ (Normalized Collision Energy ${ }^{\mathrm{TM}}$ procedure).

For each ion activation amplitude, the product ion spectra were recorded at different activation times (maximum allowed by the software: $10 \mathrm{~s}$ ). The ion dissociation time is equal to the activation time. During activation, the parent ion was held at a $q_{z}$ value of 0.250 . Each spectrum is an average of the scans acquired during four minutes. All data were acquired in the centroid mode, in order to standardize the determination of the ion intensities. For singly and doubly charged ions, the mass tolerance has been chosen so as to have one centroid peak for each isotopic peak. We measured the intensity of the monoisotopic peak only, and made a correction to account for the different isotopic patterns of parent and fragment ions. For more highly charged ions, the mass tolerance was set to a larger value, so as to have one centroid peak per species. 


\section{RESULTS AND DISCUSSION}

\section{Fragmentation pathways}

\section{Leucine enkephalin}

The resonant activation MS/MS spectra of protonated leucine enkephalin $(\mathrm{M}+\mathrm{H})^{+}$show two major fragments: $\left(\mathrm{M}+\mathrm{H}-\mathrm{H}_{2} \mathrm{O}\right)^{+}$and the $\mathrm{b}_{4}^{+}$ion. The other observed fragments $\left(\mathrm{a}_{4}^{+}, \mathrm{b}_{3}{ }^{+}\right.$and $\left.\mathrm{y}^{\prime} 3^{+}\right)$have an intensity lower than $10 \%$ of the major fragments and were not taken into account in the determination of the dissociation rate constants. In contrast, in the literature on thermal activation of leucine enkephalin ${ }^{43 ; 47}$, other fragmentation pathways have been reported, notably a very intense $\mathrm{a}_{4}^{+}$fragment ${ }^{43}$. The major difference stems from the fact that, in resonant activation, only the parent ion is activated, while the fragments rapidly cool down in the helium bath gas at room temperature. The dissociation therefore stops at the first generation of fragments, as shown in Scheme 1. In thermal activation, all ions, including the fragments, are heated, and the reaction can proceed further.

\section{Bradykinin}

MS/MS studies were carried out on three different charge states: $(\mathrm{M}+\mathrm{H})^{+},(\mathrm{M}+2 \mathrm{H})^{2+}$, and $(\mathrm{M}+3 \mathrm{H})^{3+}$. For singly protonated bradykinin, the observed fragments differ significantly from those reported by thermal activation ${ }^{44 ; 4}$, where the major fragment is the loss of $\mathrm{NH}_{3}$. Loss of water was not excluded in the quadrupole ion trap study ${ }^{48}$. Figure 1 shows the spectra obtained by resonant activation of bradykinin $(\mathrm{M}+\mathrm{H})^{+}$at two different collision energies. At low activation percentages (Fig. 1a), the loss of $\mathrm{NH}_{3}$ is the predominant fragment, but water loss can be clearly distinguished, as well as some backbone fragments $\left(\mathrm{y}_{7} 7^{+}\right.$and $\left.\mathrm{y}^{\prime \prime} 8^{+}\right)$. The proportion of water loss and of $\mathrm{y}^{\prime \prime} 8^{+}$increases when the activation percentage increases (Fig. 1b). By thermal dissociation at $200{ }^{\circ} \mathrm{C}$, backbone fragmentation was also observed ${ }^{41}$, but at different sites. For the determination of the dissociation rate constants, all fragments were taken into account. A correction was made for the intensity of $\left(\mathrm{M}+\mathrm{H}-\mathrm{NH}_{3}\right)^{+}$, taking into account the contribution of the isotopic peak of $\left(\mathrm{M}+\mathrm{H}-\mathrm{H}_{2} \mathrm{O}\right)^{+}$. For the calibration of the effective temperatures, the difference obtained if $\mathrm{y}^{\prime \prime} 7^{+}$and $\mathrm{y}^{\prime \prime} 8^{+}$are considered or not does not overcome the error 
due to the fitting and the uncertainty on the Arrhenius parameters. For the charge states $2+$ and $3+$, the fragmentation pathways observed in resonant activation MS/MS are very similar to those reported for thermal activation. $(\mathrm{M}+2 \mathrm{H})^{2+}$ fragments by water loss and by backbone fragmentation into the complementary $\mathrm{y}_{7} 7^{+}$and $\mathrm{b}_{2}{ }^{+}$ions for activation percentages $<14 \%$. At larger amplitudes (parent ion fragmentation rates $\left.>1 \mathrm{~s}^{-1}\right)$, other fragments appear: $\mathrm{y}^{\prime \prime}{ }^{+}, \mathrm{b}_{8}{ }^{+}$, and to a lesser extent $\mathrm{b}_{6}{ }^{+} .(\mathrm{M}+3 \mathrm{H})^{3+}$ fragments only by water loss at all activation energies.

\section{Ubiquitin}

In the full scan ESI-MS spectra on the quadrupole ion trap, the charge states from $5+$ to $13+$ could be detected. The Arrhenius activation parameters have been determined by BIRD for the charge states 5+ to $11+{ }^{45}$. In thermal dissociation, it has been observed that multiple water losses from the parent ion and from some of the fragments were favored for lower charge states ${ }^{41 ; 45}$. In resonant activation, we observed the loss of only one water molecule from the parent ion at the lower charge states, and no water loss from the fragments. Like in the case of leucine enkephalin dissociation, this stems from the fact that the fragments are not activated, but rapidly cool down in the trap, and do not fragment further.

We therefore restricted our study to the charge state $11+$, for which water losses are minimal in thermal activation. Typical spectra obtained at low and high activation percentages are shown in Figure 2. Most of the fragments are the same as those reported by $\mathrm{BIRD}^{45}$, but some new fragments also appear. At low activation amplitude (Fig. 2a), the fragments $\mathrm{y}^{\prime \prime} 49^{7+}$ and $\mathrm{y}_{28}{ }^{4+}$ correspond to cleavages between residues $\mathrm{Lys}^{27}-\mathrm{Ala}^{28}$ and $\mathrm{Lys}^{48}-\mathrm{Gln}^{49}$, respectively (C-terminal side of a basic lysine residue). At high activation amplitude (Fig. 2b), the fragment $\mathrm{y}^{\prime \prime} 60^{8+}$ corresponds to the cleavage between residues Glu $^{16}-\mathrm{Val}^{17}$ (C-terminal side of an acidic residue, as the other cleavages observed by BIRD). For the determination of the dissociation rate constants, all observed fragments were taken into account. 


\section{Relationship between the effective temperature and the activation amplitude}

For each ion at each activation amplitude, the dissociation rate constant is determined by linear regression of the logarithm of the fraction of parent ion as a function of the activation time, as shown for leucine enkephalin $(\mathrm{M}+\mathrm{H})^{+}$in Figure 3. The effective temperature is calculated as the temperature that, in purely thermal conditions (REX limit), would give the same dissociation rate constant as the one observed. $T_{\text {eff }}$ was determined using Equation (3),

$$
T \text { eff }=\frac{E a}{R} \cdot\left(\frac{1}{\ln A-\ln k \exp }\right)
$$

which is the Arrhenius equation modified to express the temperature as a function of the other parameters ( $E_{a}$ and $A$ are the Arrhenius parameters and $k_{\text {exp }}$ is the observed dissociation rate constant). The effective temperature is equal to the ion internal temperature only if the rapid energy exchange limit is attained. In order to stay close to the REX limit, we restricted our measurements to dissociation rate constants not greater than $5 \mathrm{~s}^{-1}$ (see further discussion below). As the maximum activation time is $10 \mathrm{~s}$ with the software used, the minimum rate constant that can be determined confidently is $\approx 0.01 \mathrm{~s}^{-1}$. The chosen thermometer molecules are leucine enkephalin $(\mathrm{M}+\mathrm{H})^{+}(\mathrm{m} / \mathrm{z}=556)$, bradykinin $(\mathrm{M}+\mathrm{H})^{+}(\mathrm{m} / \mathrm{z}=$ $1060),(\mathrm{M}+2 \mathrm{H})^{2+}(m / z=530.5),(\mathrm{M}+3 \mathrm{H})^{3+}(m / z=354)$ and ubiquitin $(\mathrm{M}+11 \mathrm{H})^{11+}(m / z=779.6)$. The published Arrhenius parameters used in the present study are listed in Table 1 together with the references. Figure $4 \mathrm{a}$ shows the results of $T_{\text {eff }}$ as a function of the activation energy expressed in $\%$, and Figure $4 \mathrm{~b}$ as a function of the actual amplitude in Volts. The abscissa are linearly related by Equation (2).

\section{Linear relationship}

The first striking result is the apparent linear relationship between the ion effective temperatures and the resonant activation amplitude (expressed either in \% or in Volts). This is in agreement with the experimental results of Goeringer et al. on leucine enkephalin from 525 to $660 \mathrm{~K}^{51}$. Figure 4a shows that this linear behavior extends to lower temperatures (down to $365 \mathrm{~K}$, corresponding to an activation 
amplitude of $7.5 \%$ ). Extrapolation at $0 \%$ activation amplitude gives an effective temperature significantly lower than room temperature (146 K instead of $300 \mathrm{~K})$. In the theoretical description of Goeringer et al. ${ }^{49 ; 50}$ (see equation (1)), which predicted a quadratic dependence of the effective temperature on the resonant activation amplitude, the curvature is marked mostly at low activation amplitudes, and the effective temperature at zero amplitude is the room temperature. A deviation from linearity is therefore expected at lower amplitudes, but could not be detected here. For the highest activation amplitudes ( $>14 \%$, which could be probed only with doubly protonated bradykinin), a deviation from linearity can be seen, but it must be pointed out that the error on these data points is much higher due to the error on the Arrhenius parameters, which are determined at lower temperatures. Moreover, as mentioned above, other fragmentation mechanisms start to appear at these high collision energies, and the apparent depletion of parent ion is overestimated, leading possibly to too high effective temperatures. Overall, the description of $T_{\text {eff }}=\mathrm{f}($ amplitude $)$ with a linear relationship is satisfactory between 365 to $600 \mathrm{~K}$.

\section{Calibration with the normalized activation amplitude (in \%)}

A striking evidence from Figure 4a is that all the ions show nearly the same dependency function of the effective temperature on the activation amplitude, when the latter is expressed in \%. Such correlation could be expected, as the Normalized Collision Energy ${ }^{\mathrm{TM}}$ procedure ensures an empirical correction for the $\mathrm{m} / \mathrm{z}$ dependence of the dissociation efficiency ${ }^{52}$. Only one set of datapoints significantly deviates compared to the others. The effective temperature of bradykinin $(\mathrm{M}+2 \mathrm{H})^{2+}$, when determined from the quadrupole ion trap thermal heating parameters ${ }^{48}$, is $15 \%$ lower than for the other ions. However, when determined from the BIRD parameters ${ }^{44}$ with the same values of $k_{\exp }$, all datapoints fit with the others. In the present case, although both sets of Arrhenius parameters agree within the reported error, the BIRD parameters should be preferred. This indicates how sensitive the method is on the accuracy of the Arrhenius parameters of the thermometer ions. For bradykinin 
$(\mathrm{M}+\mathrm{H})^{+}$, however, both sets of Arrhenius parameters give the same effective temperature within experimental error.

For the dissociation of leucine enkephalin, unexpected complications are revealed. A sudden jump in the rate constant is observed at activation amplitudes between $12 \%$ and $12.5 \%$ (see also Figure 3 ), the corresponding effective temperatures being 480 and $504 \mathrm{~K}$ respectively. Replicate experiments show that this sudden increase of the dissociation rate constant is reproducible. This may indicate that a different dissociation pathway becomes predominant at higher temperatures, although the same fragments are produced. The Arrhenius plots (see below) indicate that the two reaction pathways differ mainly by their activation energy. This interesting observation needs further investigation, but a thorough discussion is beyond the scope of the present paper. The two leucine enkephalin datasets (one with BIRD, one with QIT parameters) were not included in the calibration of the effective temperatures.

A unique calibration can be derived for all the ions, using the datapoints of bradykinin $(\mathrm{M}+\mathrm{H})^{+}($one set with the BIRD, one set with the QIT parameters), bradykinin $(\mathrm{M}+2 \mathrm{H})^{2+}(\mathrm{BIRD}$ parameters only), bradykinin $(\mathrm{M}+3 \mathrm{H})^{3+}(\mathrm{QIT}$ parameters $)$, and ubiquitin $(\mathrm{M}+11 \mathrm{H})^{11+}(\mathrm{BIRD}$ parameters $)$. The calibration line $\left(\mathrm{R}^{2}=0.994\right)$ is given by:

$$
T_{\text {eff }}(/ \mathrm{K})=(146.0 \pm 4.4)+(29.42 \pm 0.36) * \text { Ampl }(/ \%)
$$

In order to test how confidently Equation (4) can be used to calibrate the ion temperature, we determined backwards the Arrhenius parameters from the measured dissociation rate constants at different Ampl (/\%), the temperature being calculated from Ampl (/\%) with Equation (4). If this equation is valid for all ions whatever their $\mathrm{m} / \mathrm{z}$, the Arrhenius parameters should be equal to the published values used for the calibration. This backwards calculation is therefore used as a "quality check" of the calibration. The results are shown in Table 2. A perfect agreement is found only for the bradykinin $(\mathrm{M}+2 \mathrm{H})^{2+}$ ion. For the other ions, although the values often agree within experimental error, the agreement is not satisfactory for the purpose of a universal calibration of the effective temperatures (since the values in Table 1 have been used to determine the effective temperatures, exactly the same values should be found in Table 2). This prompted us to search for an improved calibration of the 
effective temperature, with a better correction of the effect of the ion mass-to-charge ratio. Furthermore, as not all quadrupole ion trap instruments use the normalized collision energy procedure, a more general equation relating the effective temperature to the actual resonant activation amplitude (in Volts) was sought.

\section{Calibration with the actual activation amplitude (in Volts) and dependency on $\mathrm{m} / \mathrm{z}$}

The starting point is the series of thermometer ions for which the effective temperatures have been determined as a function of the activation amplitude (Figure $4 \mathrm{~b}$ ). We have shown above that $T_{\text {eff }}$ is related to Ampl(/\%) by a linear relationship in the range $[365-600 \mathrm{~K}]$. With the change of abcissa given by equation (2), the relationship between $T_{\text {eff }}$ and $A m p l(/ V)$ is still linear, but the slope and intercept are now different for each ion. For each ion the effective temperature can be written as:

$$
T_{\text {eff }}(/ \mathrm{K})=\text { intercept }+ \text { slope } * \text { Ampl (/V) }
$$

The $m / z$ dependencies of the slope and intercept are shown in Figure 5 (a) and (b) respectively. The points corresponding to leucine enkephalin (gray) and the point corresponding to bradykinin $(\mathrm{M}+2 \mathrm{H})^{2+}$ with QIT parameters (white) are shown in Figure 5 for information, bur were not taken into account for the fittings. The slope does not depend linearly on $\mathrm{m} / \mathrm{z}$. Rather, the best fitting is found using:

$$
\text { slope }=1 /\left[\mathrm{a}+\mathrm{b}^{*}(m / z)\right]
$$

The intercept is found to depend weakly, but significantly on $\mathrm{m} / \mathrm{z}$ :

$$
\text { intercept }=\mathrm{INT}+\mathrm{c}^{*}(\mathrm{~m} / \mathrm{z})
$$

Equations (6) and (7) can be introduced in equation (5) to give:

$$
T_{\text {eff }}(/ \mathrm{K})=\mathrm{INT}+\mathrm{c}^{*}(m / z)+\frac{A m p l(/ V)}{a+b^{*}(m / z)}
$$

Equation (8) can be used to calculate the effective temperature of any ion from its mass-to-charge ratio and the applied activation amplitude. It is valid over the calibration range ( $T_{\text {eff }}$ from 365 to $600 \mathrm{~K}$ ). At lower temperatures, a deviation from linearity is expected and equation (8) should not be used. At higher temperatures, the validity rather depends on whether the REX limit is still reached or not (see below). The most accurate results will be obtained for ion $\mathrm{m} / \mathrm{z}$ values within the calibration range (from 
354 to $1060 \mathrm{Th}$ in the present case), but the equation is likely to be valid on a broader range. For a given instrument, a, b, c and INT can be determined by a calibration with bradykinin (charge states $1+, 2+$ and $3+$ ) and ubiquitin (charge state 11+) as described above. For the instrument used in the present study, we found $\mathrm{a}=(2.84 \pm 0.4) 10^{-4}$ Volt.K $\mathrm{K}^{-1}, \mathrm{~b}=(2.61 \pm 0.09) 10^{-6} \mathrm{Vollts}^{-1} \cdot(\mathrm{m} / \mathrm{z})^{-1}, \mathrm{c}=0.055 \pm 0.007$ K. $(\mathrm{m} / \mathrm{z})^{-1}$, and INT $=116.1 \pm 5.5 \mathrm{~K}$. The calibration is likely to be influenced by the bath gas temperature, the bath gas pressure, the nature of the bath gas, and the parent ion $q z$ value.

\section{Determination of Arrhenius activation parameters}

To test the validity of equation (8) and the adequacy of the parameters a, b, c and INT found by the calibration, the Arrhenius parameters were determined again using the measured dissociation rate constants and the effective temperatures determined with equation (8). The results of the fittings are shown in the supporting information, and the Arrhenius parameters are summarized in Table 3. Compared to Table 2, we can see that, while the Arrhenius parameters have not changed for leucine enkephalin $(\mathrm{M}+\mathrm{H})^{+}(\mathrm{m} / \mathrm{z} 556)$ and bradykinin $(\mathrm{M}+2 \mathrm{H})^{2+}(\mathrm{m} / \mathrm{z} 530.5)$, the parameters calculated using equation (8) show a significant improvement compared to equation (4) for the ions bradykinin $(\mathrm{M}+\mathrm{H})^{+}$ $\left(m / z\right.$ 1060), ubiquitin $(\mathrm{M}+11 \mathrm{H})^{11+}\left(\mathrm{m} / \mathrm{z}\right.$ 779.6) and bradykinin $(\mathrm{M}+3 \mathrm{H})^{3+}(\mathrm{m} / \mathrm{z} 334)$. The Arrhenius parameters are now in close agreement with the published value on the entire $m / z$ range. Using the approximate equation (4) gives good results only for $m / z$ close to $\approx 540 \mathrm{Th}$. Using equation (8) instead, the first requirement for the determination of Arrhenius parameters of unknowns, namely the calibration of the ion effective temperatures as a function of the activation amplitude and the ion $\mathrm{m} / \mathrm{z}$, is satisfactorily met.

There is a second requirement for the so-determined Arrhenius parameters to be meaningful: the rapid energy exchange limit must be attained. This means that ion activation and deactivation rates must be larger than the dissociation rate, so that the ion internal energy distribution is a Boltzmann distribution. In blackbody infrared activation, it has been well documented that the ion activation and dissociation rates increase with the ion $\operatorname{size}^{46}$. The situation is different in ion trap resonant activation, as ion 
activation and deactivation are due to collisions. Goeringer and McLuckey have reported modeling of

collisional cooling under typical ion trap storage (not resonant activation) conditions ${ }^{53}$. The apparent ion cooling rates were shown to decrease with the ion size. This means that reaching the REX limit is more likely to be achieved for small ions than for large ones, at fixed dissociation rate. At $450 \mathrm{~K}$, the ion apparent cooling rate constants are between 530 and $1700 \mathrm{~s}^{-1}$ for the model peptide (AG)8 (1043 Da) and between 290 and $960 \mathrm{~s}^{-1}$ for $(\mathrm{AG})_{32}(4118 \mathrm{Da})$. In the present study, we limited our experiments to dissociation rate constants $k_{o b s}<5 \mathrm{~s}^{-1}$. The excellent agreement with the published Arrhenius parameters and those recalculated using equation (8) suggests that the effective temperature approach and the empirical calibration described here is satisfactory, at least for ion masses up to $8500 \mathrm{Da}$. Nevertheless, detailed modeling would be required to assess (1) whether the ion internal energy distribution is identical to a Boltzmann distribution, and (2) what is are the maximum dissociation rates to ensure be in the REX limit.

\section{Improved normalization of the collision energy}

Given the fact that the normalization of the collision energies using equation (2) is an ampirical calibration based on the fragmentation efficiencies of some test compounds ${ }^{52}$, the superimposing of the different lines in Figure 4a is already impressive. Actually, the criteria of "achieving similar fragmentation efficiencies" for a given experimental setting, could be replaced by the criteria of "achieving the same effective temperature". The normalized collision energy parameter used by default in the LCQ instrument is obtained by rearrangement of Equation (2):

$$
\operatorname{Ampl}(/ \%)=\frac{A m p l(/ V) * 30}{0.4+0.002 *(m / z)}
$$

where the pencentage scale is actually an arbitrary unit scale. Using equation (8), a new reduced parameter, having the dimensions of a temperature, can be defined to more accurately describe the exact dependency of the ion effective temperature on the activation amplitude and on $\mathrm{m} / \mathrm{z}$ :

$$
T_{e f f \text { norm }}(/ \mathrm{K})=\mathrm{c}^{*}(m / z)+\frac{A m p l(/ V)}{a+b^{*}(m / z)}
$$


The comparison between Equations (9) and (10) emphasizes (i) that the Normalized Collision Energy $^{\mathrm{TM}}$ procedure actually corrects for the dependence of the slope of the $T_{\text {eff }} \mathrm{f}(\mathrm{Ampl}(\mathrm{V}))$ function, (ii) how the parameters of Equation (2) are related to the parameters a and b determined here, and (iii) that the improvement of the calibration comes from the correction for the dependence of the intercept on the ion $m / z$.

\section{CONCLUSIONS}

The excellent agreement between the published Arrhenius parameters and those determined with the effective temperature given by equation (8) indicates that this equation and the parameters INT, a, b and c determined from the calibration procedure described above are adequate to calculate the effective temperature of any given ion. As a consequence, the Arrhenius parameters can be determined by resonant activation in a quadrupole ion trap. This is the first example of direct and simple determination of Arrhenius parameters using collisional activation. The major advantage over thermal methods is that the calibration procedure described herein is applicable to any quadrupole ion trap instrument, without hardware modification. The measurements can be automated on most commercial instruments. The experiments are therefore more reproducible, and the errors on the Arrhenius parameters can be reduced significantly (see the errors reported in Table 1 and Tables 2/3).

The method proposed here can be applied to the determination of Arrhenius activation parameters for peptide fragmentation. Tabulated values of $E_{a}$ and $A$ as a function of the cleavage site would be extremely useful to improve sequencing algorithms, using not only the masses of the fragments, but also the probability of each fragmentation channel as a function of the experimental conditions. Another major difference between collisional activation by resonant activation and thermal activation methods is that other dissociation channels can be probed. As the fragments are not further activated in resonant excitation, consecutive neutral losses are minimized. Moreover, higher effective temperatures can be easily accessed. This is particularly interesting for non-covalent complexes. For example, in the case of DNA duplexes, neutral base loss is the major fragment at low internal energies. Duplex dissociation into 
single strands can only be achieved at higher internal energies, like those achieved by resonant activation in a quadrupole ion trap ${ }^{54}$. Quantitative studies on the dissociation of non-covalent complexes using the present method are currently underway.

ACKNOWLEDGMENT. Preliminary data from this work were presented at the $15^{\text {th }}$ Sanibel Conference on Mass Spectrometry, January 24-28, 2003, and the authors benefited from fruitful discussion on this occasion. VG is grateful to the FNRS (Fonds National de la Recherche Scientifique, Belgium) for a postdoctoral research fellowship and to the Alexander von Humboldt foundation for sponsoring a research stay in Germany. This work has been supported by the ARC (Actions de Recherche Concertées / Communauté Française de Belgique). Dr. Andrea Schmidt is acknowledged for useful comments on the manuscript.

Supporting Information Available. The Arrhenius plots which have led to the values in Tables 2 and 3 are given in Figure S1. 


\section{FIGURES.}
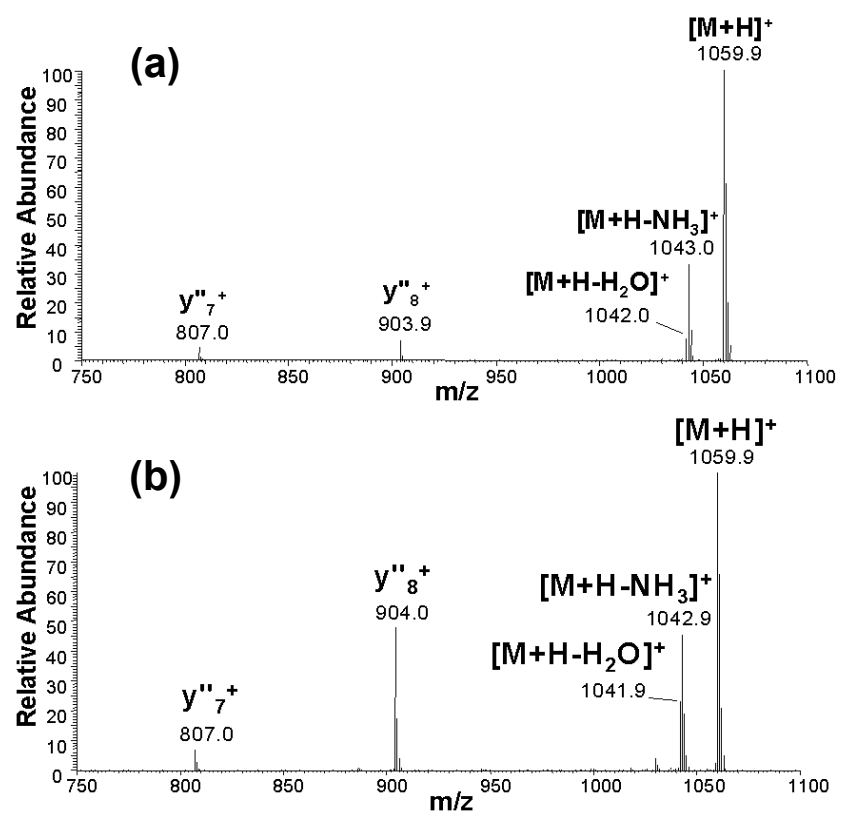

Figure 1. Resonant activation MS/MS spectra of singly protonated bradykinin at (a) activation amplitude $11 \%$ and activation time $10 \mathrm{~s}$ and (b) activation amplitude $14 \%$ and activation time $200 \mathrm{~ms}$. 

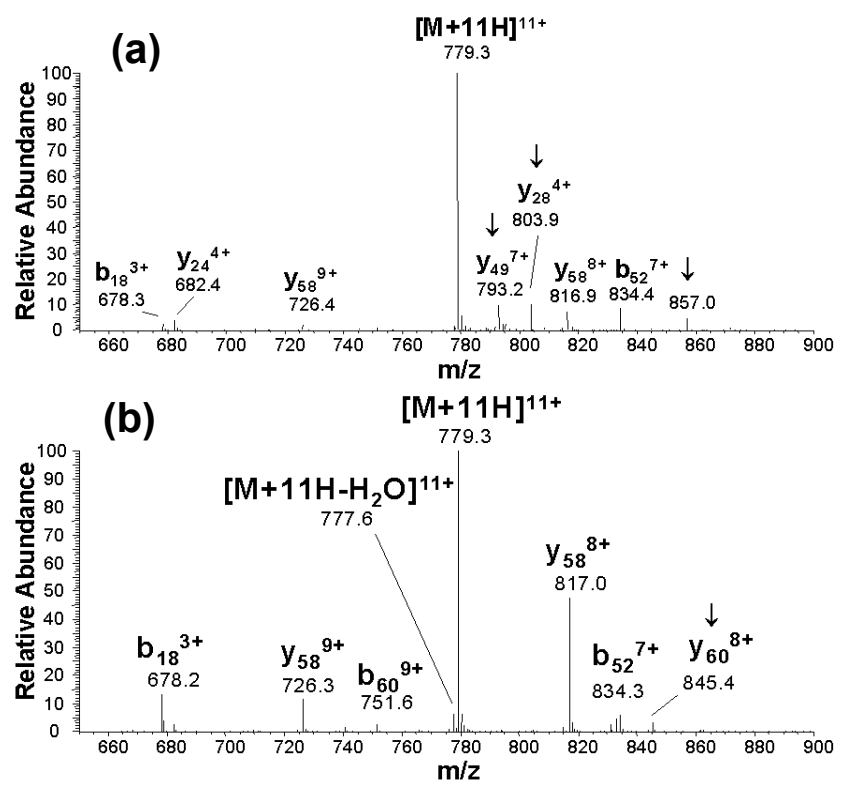

Figure 2. Resonant activation $\mathrm{MS} / \mathrm{MS}$ spectra of ubiquitin $(\mathrm{M}+11 \mathrm{H})^{11+}$ at (a) activation amplitude $9.5 \%$ and activation time $10 \mathrm{~s}$ and (b) activation amplitude $12 \%$ and activation time $75 \mathrm{~ms}$. The arrows indicate the fragments which have not been reported by BIRD ${ }^{45}$. 


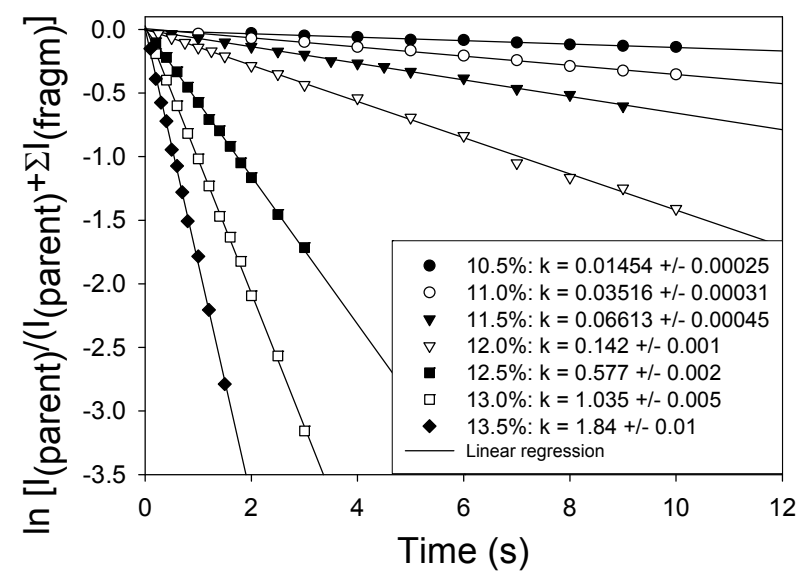

Figure 3. Dissociation rate constants of singly protonated leucine enkephalin as a function of the resonant activation amplitude. Data fit to pseudo-first order kinetics. 

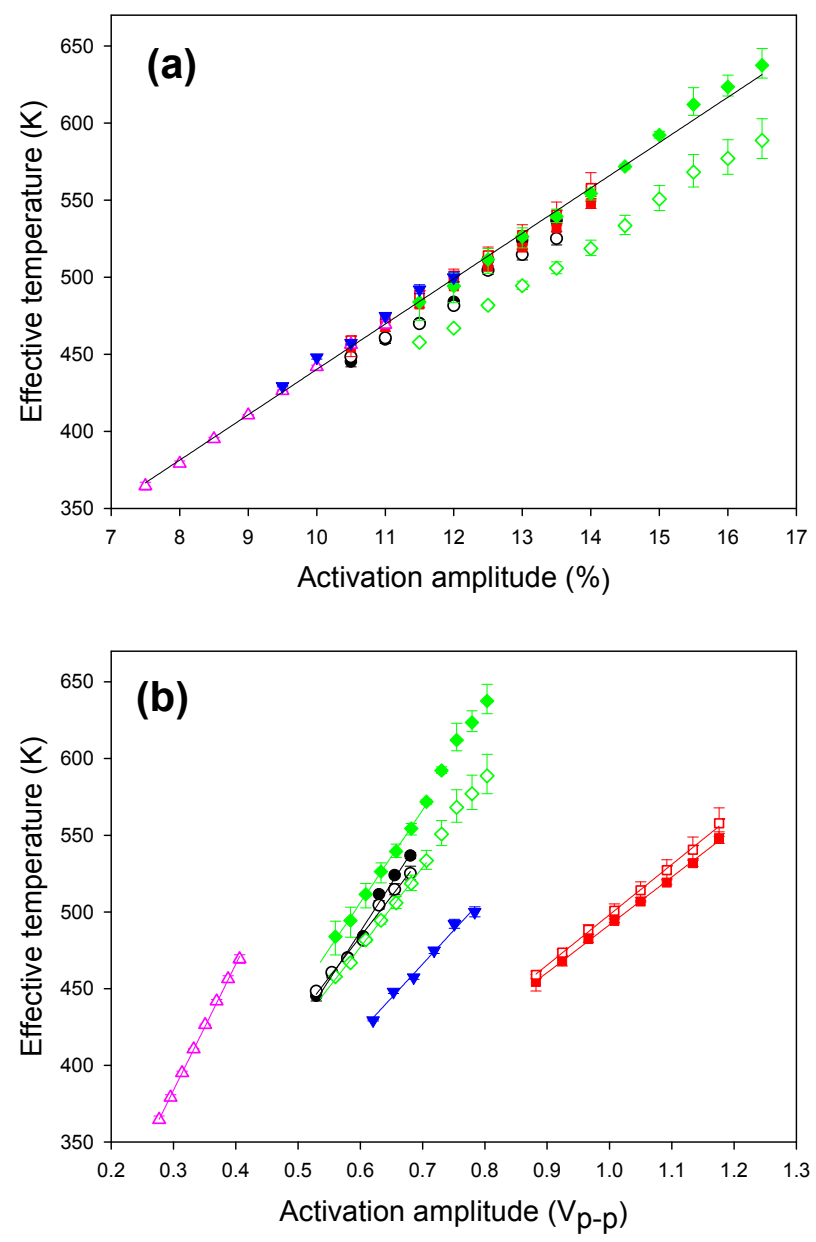

Figure 4. Effective temperature as a function of the resonant activation amplitude expressed in \% (a) and in Volts (b). The Arrhenius parameters used for the calculation of $T_{\text {eff }}$ are summarized in Table 1. Black circles: leucine enkephalin $(\mathrm{M}+\mathrm{H})^{+}$; red squares: bradykinin $(\mathrm{M}+\mathrm{H})^{+}$; green diamonds: bradykinin $(\mathrm{M}+2 \mathrm{H})^{2+}$; pink triangles up: bradykinin $(\mathrm{M}+3 \mathrm{H})^{3+}$; blue triangles down: ubiquitin $(\mathrm{M}+11 \mathrm{H})^{11+}$. Full symbols indicate the use of BIRD parameters, and open symbols indicate the use of QIT parameters. The error bars take into account both the error on the determination of $k_{\exp }$ by linear regression, and the error on the published Arrhenius parameters. 

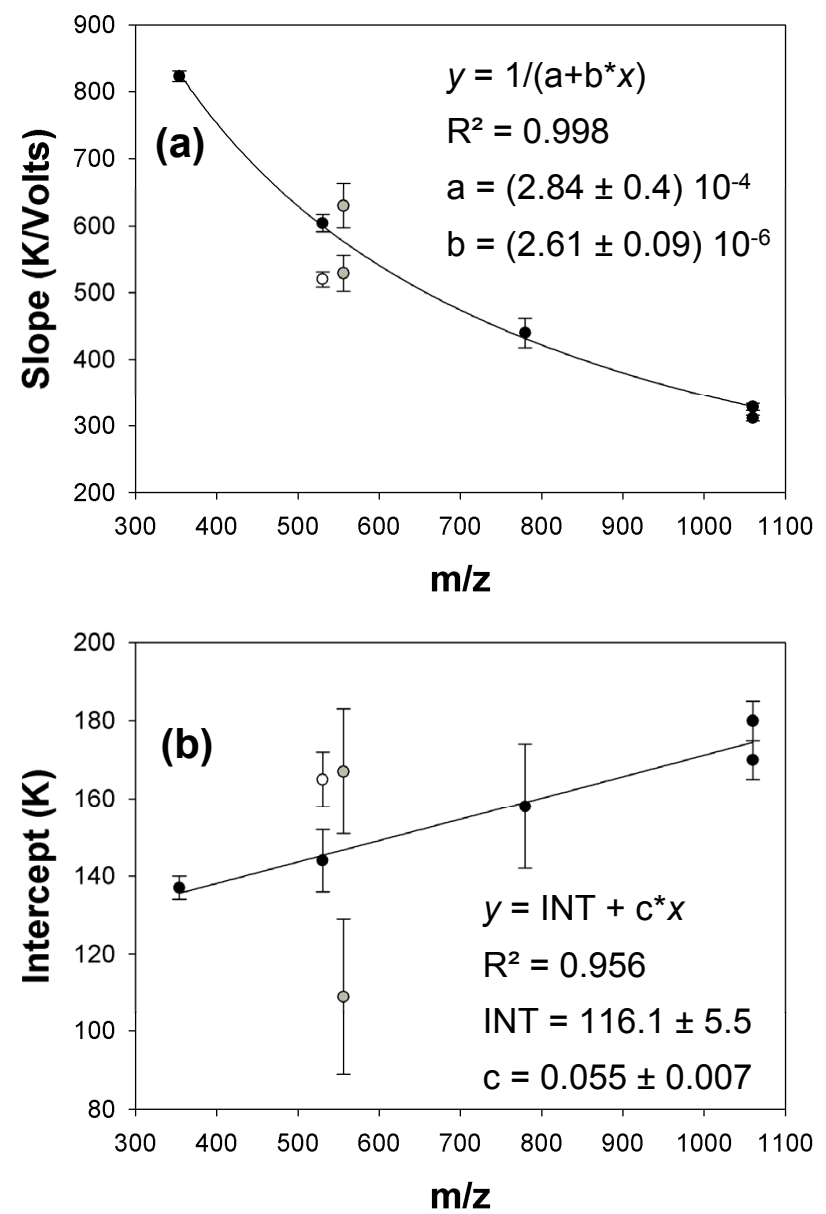

Figure 5. Fitting of the $m / z$ dependence of the slope (a) and intercept (b) of the linear regressions shown in Figure $4 \mathrm{~b}$ (effective temperatures as a function of the actual activation amplitude). The white circle corresponds to bradykinin $(\mathrm{M}+2 \mathrm{H})^{2+}$ for which $T_{\text {eff }}$ was determined using the QIT parameters. The gray circles correspond to protonated leucine enkephalin. These points were left out for the calibration. The error bars correspond to the error of the linear least squares fitting of the data in Figure $4 \mathrm{~b}$ using Equation (5). 
SCHEME.

Scheme 1.

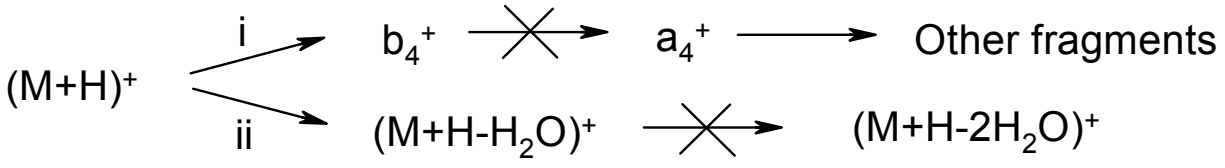


TABLES.

Table 1. Published Arrhenius parameters of the thermometer ions used in the present study.

\begin{tabular}{|l|l|l|l|l|l|}
\hline & & BIRD & ref & QIT & ref \\
\hline Leucine enkephalin $(\mathrm{M}+\mathrm{H})^{+}$ & $\log A:$ & $10.5 \pm 0.6$ & 43 & $12.55 \pm 0.87$ & 47 \\
& $E_{a}(\mathrm{eV}):$ & $1.09 \pm 0.06$ & & $1.28 \pm 0.08$ & \\
\hline Bradykinin $(\mathrm{M}+\mathrm{H})^{+}$ & $\log A:$ & $12.59^{\mathrm{a}}$ & 44 & $12.12 \pm 1.0$ & 48 \\
& $E_{a}(\mathrm{eV}):$ & $1.3^{\mathrm{b}}$ & & $1.27 \pm 0.09$ & \\
\hline Bradykinin $(\mathrm{M}+2 \mathrm{H})^{2+}$ & $\log A:$ & $6.94^{\mathrm{a}}$ & 44 & $7.63 \pm 0.67$ & 48 \\
& $E_{a}(\mathrm{eV}):$ & $0.8^{\mathrm{b}}$ & & $0.82 \pm 0.06$ & \\
\hline Bradykinin $(\mathrm{M}+3 \mathrm{H})^{3+}$ & $\log A:$ & - & & $9.3 \pm 0.36$ & 48 \\
& $E_{a}(\mathrm{eV}):$ & & & $0.79 \pm 0.03$ & \\
\hline Ubiquitin $(\mathrm{M}+11 \mathrm{H})^{11+}$ & $\log A:$ & $16.7 \pm 0.7$ & 45 & - & \\
& $E_{a}(\mathrm{eV}):$ & $1.55 \pm 0.06$ & & & \\
\hline
\end{tabular}

${ }^{a}$ Error was not given by the authors. For the estimation of error bars of $T_{\text {eff, }}$, we assumed an error of $10 \%$.

${ }^{\mathrm{b}}$ Error was not given for each measurement. For the estimation of error bars of $T_{\text {eff }}$, we assumed an error of $0.1 \mathrm{eV}$ (the maximal error according to the authors ${ }^{44}$ ). 
Table 2. Arrhenius parameters determined by resonant activation with the calibration of the effective temperatures using equation (4). The errors are those associated with the linear least squares regression.

\begin{tabular}{|l|l|l|}
\hline Ion & \multicolumn{2}{|l|}{ Arrhenius parameters } \\
\hline Leucine enkephalin $(\mathrm{M}+\mathrm{H})^{+}$ & $\log A:$ & $9.2 \pm 0.4^{\mathrm{a}}$ \\
& $E_{a}(\mathrm{eV}):$ & $1.00 \pm 0.05^{\mathrm{a}}$ \\
& $\log A:$ & $9.08 \pm 0.09^{\mathrm{b}}$ \\
& $E_{a}(\mathrm{eV}):$ & $0.95 \pm 0.01^{\mathrm{b}}$ \\
\hline Bradykinin $(\mathrm{M}+\mathrm{H})^{+}$ & $\log A:$ & $11.25 \pm 0.17$ \\
& $E_{a}(\mathrm{eV}):$ & $1.18 \pm 0.02$ \\
\hline Bradykinin $(\mathrm{M}+2 \mathrm{H})^{2+}$ & $\log A:$ & $6.91 \pm 0.18$ \\
& $E_{a}(\mathrm{eV}):$ & $0.80 \pm 0.02$ \\
\hline Bradykinin $(\mathrm{M}+3 \mathrm{H})^{3+}$ & $\log A:$ & $9.64 \pm 0.09$ \\
& $E_{a}(\mathrm{eV}):$ & $0.819 \pm 0.008$ \\
\hline Ubiquitin $(\mathrm{M}+11 \mathrm{H})^{11+}$ & $\log A:$ & $16.2 \pm 0.8$ \\
& $E_{a}(\mathrm{eV}):$ & $1.49 \pm 0.07$ \\
\hline
\end{tabular}

${ }^{\mathrm{a}}$ Low-temperature process.

${ }^{\mathrm{b}}$ High-temperature process. 
Table 3. Arrhenius parameters determined by resonant activation with the calibration of the effective temperatures using equation (8). The errors are those associated with the linear least squares regression.

\begin{tabular}{|l|l|l|}
\hline Ion & \multicolumn{2}{|l|}{ Arrhenius parameters } \\
\hline Leucine enkephalin $(\mathrm{M}+\mathrm{H})^{+}$ & $\log A:$ & $9.25 \pm 0.4^{\mathrm{a}}$ \\
& $E_{a}(\mathrm{eV}):$ & $0.99 \pm 0.04^{\mathrm{a}}$ \\
& $\log A:$ & $9.12 \pm 0.09^{\mathrm{b}}$ \\
& $E_{a}(\mathrm{eV}):$ & $0.945 \pm 0.01^{\mathrm{b}}$ \\
\hline Bradykinin $(\mathrm{M}+\mathrm{H})^{+}$ & $\log A:$ & $12.20 \pm 0.17$ \\
& $E_{a}(\mathrm{eV}):$ & $1.29 \pm 0.02$ \\
\hline Bradykinin $(\mathrm{M}+2 \mathrm{H})^{2+}$ & $\log A:$ & $6.91 \pm 0.17$ \\
& $E_{a}(\mathrm{eV}):$ & $0.79 \pm 0.02$ \\
\hline Bradykinin $(\mathrm{M}+3 \mathrm{H})^{3+}$ & $\log A:$ & $9.27 \pm 0.09$ \\
& $E_{a}(\mathrm{eV}):$ & $0.788 \pm 0.007$ \\
\hline Ubiquitin $(\mathrm{M}+11 \mathrm{H})^{11+}$ & $\log A:$ & $16.94 \pm 0.83$ \\
& $E_{a}(\mathrm{eV}):$ & $1.555 \pm 0.08$ \\
\hline
\end{tabular}

${ }^{\text {a }}$ Low-temperature process.

${ }^{\mathrm{b}}$ High-temperature process. 


\section{REFERENCES.}

(1) Fenn, J. B.; Mann, M.; Meng, C. K.; Wong, S. F.; Whitehouse, C. M. Science 1989, 246, 64-71.

(2) Fenn, J. B.; Mann, M.; Meng, C. K.; Wong, S. F. Mass Spectrom. Rev. 1990, 9, 37-70.

(3) Mann, M. Org. Mass Spectrom. 1990, 25, 575-587.

(4) Smith, R. D.; Light-Wahl, K. J. Biol. Mass Spectrom. 1993, 22, 493-501.

(5) Przybylski, M.; Glocker, M. O. Angew. Chem. Int. Ed. 1996, 35, 806-826.

(6) Smith, R. D.; Bruce, J. E.; Wu, Q.; Lei, Q. P. Chem. Soc. Rev. 1997, 26, 191-202.

(7) Loo, J. A. Mass Spectrom. Rev. 1997, 16, 1-23.

(8) Hofstadler, S. A.; Griffey, R. H. Chem. Rev. 2001, 101, 377-390.

(9) Schalley, C. A. Mass Spectrom. Rev. 2001, 20, 253-309.

(10) Hernandez, H.; Robinson, C. V. J. Biol. Chem. 2001, 276, 46685-46688.

(11) Rostom, A. A.; Tame, J. R. H.; Ladbury, J. E.; Robinson, C. V. J. Mol. Biol. 2000, 296, 269-279.

(12) Nesatyy, V. J. Mass Spectrom. 2001, 36, 950-959.

(13) Hunter, C. L.; Mauk, A. G.; Douglas, D. J. Biochemistry 2000, 36, 1018-1025.

(14) Gross, D. S.; Zhao, Y.; Williams, E. R. J. Am. Soc. Mass Spectrom. 1997, 8, 519-524.

(15) Felitsyn, N.; Kitova, E. N.; Klassen, J. S. Anal. Chem. 2001, 73, 4647-4661.

(16) Lee, S.; Wyttenbach, T.; von Helden, G.; Bowers, M. T. J. Am. Chem. Soc. 1995, 117, 10 159-10 160. 
(17) Kaleta, D. T.; Jarrold, M. F. J. Am. Chem. Soc. 2002, 124, 1154-1155.

(18) Lorenz, S. A.; Maziarz III, E. P.; Wood, T. D. J. Am. Soc. Mass Spectrom. 2001, 12, 795804.

(19) Yamada, N.; Suzuki, E.-I.; Hirayama, K. Rapid Commun. Mass Spectrom. 2002, 16, 293299.

(20) Badman, E. R.; Chrisman, P. A.; McLuckey, S. A. Anal. Chem. 2002, 74, 6237-6243.

(21) Vékey, K. J. Mass Spectrom. 1996, 31, 445-463.

(22) Ervin, K. M. Chem. Rev. 2001, 101, 391-444.

(23) Armentrout, P. B.; Baer, T. J. Phys. Chem. 1996, 100, 12866-12877.

(24) Rodgers, M. T.; Armentrout, P. B. Mass Spectrom. Rev. 2000, 19, 215-247.

(25) Armentrout, P. B. J. Am. Soc. Mass Spectrom. 2002, 13, 419-434.

(26) More, M. B.; Ray, D.; Armentrout, P. B. J. Am. Chem. Soc. 1999, 121, 417-423.

(27) Armentrout, P. B. Int. J. Mass Spectrom. 1999, 193, 227-240.

(28) McLuckey, S. A.; Goeringer, D. E. J. Mass Spectrom. 1997, 32, 461-474.

(29) Schweikhard, L.; Marshall, A. G. J. Am. Soc. Mass Spectrom. 1993, 4, 433-452.

(30) Sobott, F.; Hernandez, H.; McCammon, M. G.; Tito, M. A.; Robinson, C. V. Anal. Chem. 2002, 74, 1402-1407.

(31) Rostom, A. A.; Fucini, P.; Benjamin, D. R.; Juenemann, R.; Nierhaus, K. H.; Hartl, F. U.; Dobson, C. M.; Robinson, C. V. Proc. Natl. Acad. Sci. USA 2000, 97, 5185-5190.

(32) Laskin, J.; Byrd, M.; Futrell, J. H. Int. J. Mass Spectrom. 2000, 195/196, 285-302. 
(33) Laskin, J.; Futrell, J. H. J. Phys. Chem. A 2000, 104, 5484-5494.

(34) Douglas, D. J. J. Am. Soc. Mass Spectrom. 1998, 9, 101-113.

(35) Schnier, P. D.; Jurchen, J. C.; Williams, E. R. J. Phys. Chem. B 1999, 103, 737-745.

(36) McLuckey, S. A.; Wells, J. M.; Stephenson, J. L., Jr.; Goeringer, D. E. Int. J. Mass Spectrom. 2000, 200, 137-161.

(37) Nesatyy, V. J.; Laskin, J. Int. J. Mass Spectrom. 2002, 221, 245-262.

(38) Dunbar, R. C. J. Phys. Chem. 1994, 98, 8705-8712.

(39) Dunbar, R. C.; McMahon, T. B.; Thölmann, D.; Tonner, D. S.; Salahub, D. R.; Wei, D. J. Am. Chem. Soc. 1995, 117, 12819-12825.

(40) Dunbar, R. C.; McMahon, T. B. Science 1998, 279, 194-197.

(41) Price, W. D.; Schnier, P. D.; Williams, E. R. Anal. Chem. 1996, 68, 859-866.

(42) Price, W. D.; Schnier, P. D.; Jockusch, R. A.; Strittmatter, E. F.; Williams, E. R. J. Am. Chem. Soc. 1996, 118, 10640-10644.

(43) Schnier, P. D.; Price, W. D.; Strittmatter, E. F.; Williams, E. R. J. Am. Soc. Mass Spectrom. 1997, 8, 771-780.

(44) Schnier, P. D.; Price, W. D.; Jockusch, R. A.; Williams, E. R. J. Am. Chem. Soc. 1996, $118,7178-7189$.

(45) Jockusch, R. A.; Schnier, P. D.; Price, W. D.; Strittmatter, E. F.; Demirev, P. A.; Williams, E. R. Anal. Chem. 1997, 69, 1119-1126.

(46) Price, W. D.; Williams, E. R. J. Phys. Chem. A 1997, 101, 8844-8852. 
(47) Asano, K. G.; Goeringer, D. E.; McLuckey, S. A. Int. J. Mass Spectrom. 1999, 185/186/187, 207-219.

(48) Butcher, D. J.; Asano, K. G.; Goeringer, D. E.; McLuckey, S. A. J. Phys. Chem. A 1999, $103,8664-8671$

(49) Goeringer, D. E.; McLuckey, S. A. J. Chem. Phys. 1996, 104, 2214-2221.

(50) Goeringer, D. E.; McLuckey, S. A. Rapid Commun. Mass Spectrom. 1996, 10, 328-334.

(51) Goeringer, D. E.; Asano, K. G.; McLuckey, S. A. Int. J. Mass Spectrom. 1999, 182/183, $275-288$.

(52) Lopez, L. L.; Tiller, P. R.; Senko, M. W.; Schwartz, J. C. Rapid Commun. Mass Spectrom. 1999, 13, 663-668.

(53) Goeringer, D. E.; McLuckey, S. A. Int. J. Mass Spectrom. Ion. Proc. 1998, 177, 163-174.

(54) Gabelica, V.; De Pauw, E. J. Am. Soc. Mass Spectrom. 2002, 13, 91-98. 